\title{
The Effect of Beam Scan Strategies on the Microstructure of EBM Additively Manufactured Inconel 738
}

Chris Blackwell ${ }^{1}$, Meiyue Shao $^{2}$, Sriram Vijayan ${ }^{2}$, Sabina Kumar ${ }^{3}$, Sudarsanam Babu ${ }^{3}$ and Joerg Jinschek $^{2}$

${ }^{1}$ The Ohio State University, St. Clairsville, Ohio, United States, ${ }^{2}$ The Ohio State University, Columbus, Ohio, United States, ${ }^{3}$ The University of Tennessee, Knoxville, Knoxville, Tennessee, United States

Electron beam melting (EBM) is a form of additive manufacturing (AM) that offers fast, customizable, near-net production of metallic parts by depositing material "layer by layer." In EBM, an electron beam scans across a bed of metal powder, thereby melting the powder in the shape of a desired part. The melted powder solidifies into a layer, the layer is covered with a new layer of powder, and the process repeats. Consequently, any location within the build could be rapidly heated above solid-solid phase transition temperatures several times as the beam scans the layer(s) above [1]. These beam-dependent, cyclic thermal profiles are known to strongly affect morphology and microstructure, resulting in anisotropic grain sizes, macro- and micro-porosity, and complex phase morphologies [2,3]. This directly impacts the mechanical properties of AM-built parts. While these complex thermal profiles can be controlled with beam scanning strategies, they are not fully understood. Understanding and controlling AM thermal gradients in space and time opens the opportunity to design unique, tailored, desirable microstructures.

Here, we characterized the microstructural variations in an EBM Inconel 738 build printed under welldefined conditions using a random EBM scanning pattern. This will act as the baseline for comparison with variations in microstructures when using other EBM scan strategies, such as the standard raster scan method. The microstructure of our AM build, a 15x15x25mm block, was systematically investigated in build direction and in build plane. The AM block was therefore cut at the bottom and the top and along the side. Standard mechanical polishing procedures were used. Each sample surface was then characterized at nine specific locations across the sample surface to ensure statistically significant data sets.

Our systematic characterization experiments included optical microscopy (OM), scanning electron microscopy (SEM), and site-specific microhardness testing. OM yielded quantities for macro-pore size, distribution, and shape. SEM resulted in high-contrast images which were segmented as shown in Figure 1 to quantify $\gamma / \gamma^{\prime}$ size and aspect ratio, $\gamma^{\prime}$ volume fraction, carbide morphology, and carbide distribution. Additionally, SEM images were taken using T1, T2, and T3 Trinity detectors on Thermo Scientific's Apreo SEM (shown in Figure 2). While the $\gamma^{\prime}$ volume fraction was found to be constant in the build direction, the size of the individual $\gamma^{\prime}$ precipitates decreased by $30 \%$ from bottom to top. Subsequent Vickers microhardness measurements indicated a slight increase of hardness at the top of the build. This corresponds to the decrease in size of $\gamma^{\prime}$ precipitates. Throughout the sample, pores remained circular with a median diameter of $9.3 \mu \mathrm{m}$ (standard deviation $6.1 \mu \mathrm{m}$ ) and a total area fraction of $0.1 \%$. 


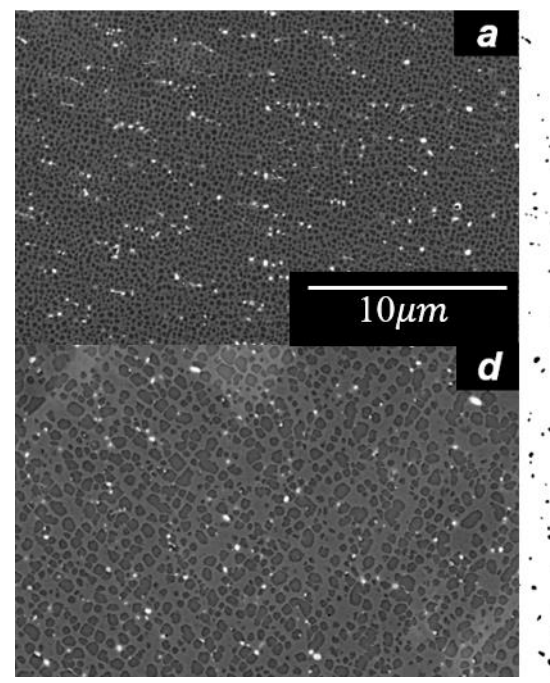

\section{b}
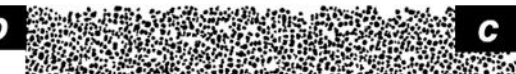

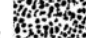

W
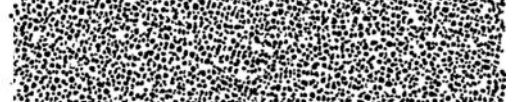

放

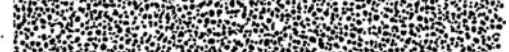

$e$

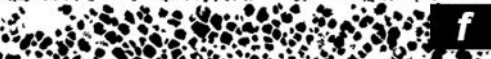

$+3$

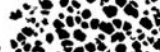

(2)
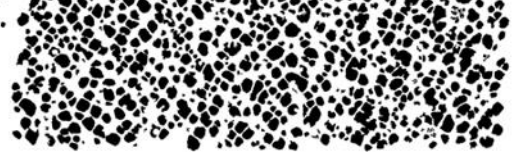

Figure 1. SEM backscattered electron (BSE) images of IN 738 in the build direction taken with an annular BSE detector. (a) is an original SEM-BSE image taken near the top of the build. (d) was taken near the bottom. Using MIPAR the SEM-BSE images in (a) and (d) can be segmented in order to enhance contrast and isolate carbides (in (b) and (e) and $\gamma^{\prime}$ precipitates (in (c) and (f)) for further statistical data analyses.

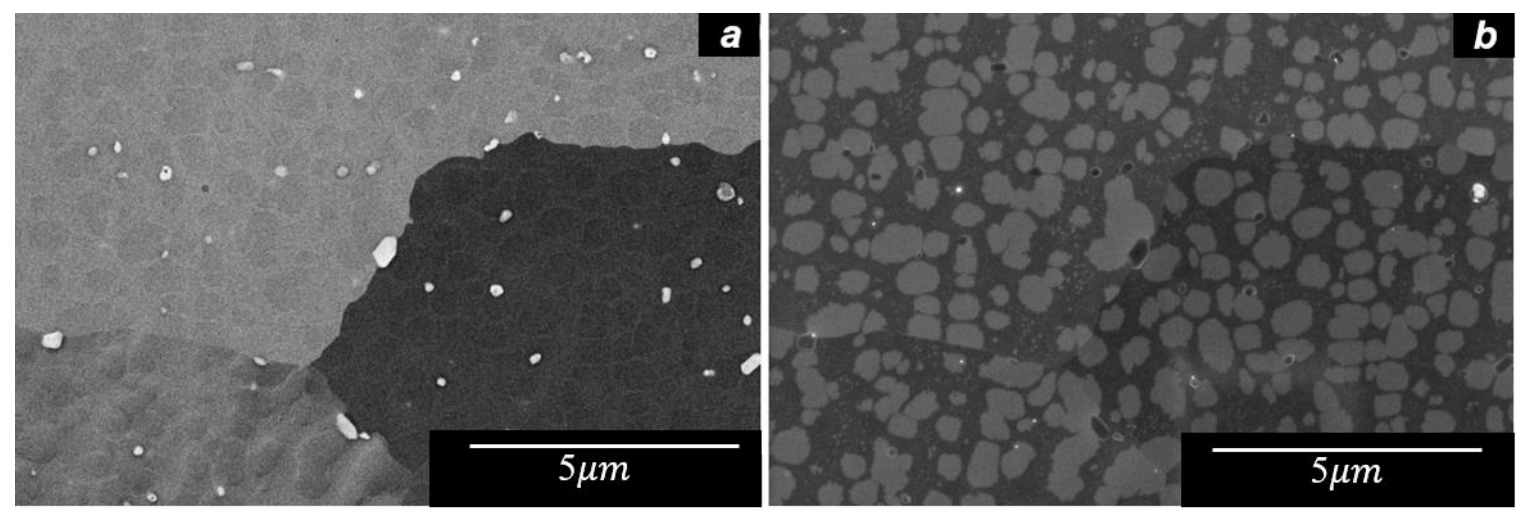

Figure 2. SEM images of IN 738 in the build plane near the bottom of the build. (a) The image taken with the T1 detector shows strong elemental contrast and channeling contrast. (b) At the same location, the image taken with the T3 detector shows extreme surface sensitivity that reveals only the $\gamma^{\prime}$ precipitates.

\section{References}

[1] W.J. Sames, F.A. List, S. Pannala, R.R. Dehoff, and S. S. B. The Metallurgy and Processing Science of Metal Additive Manufacturing. Int. Mater. Rev. 61, 315-360 (2016).

[2] Raghavan, N. et al. Numerical modeling of heat-transfer and the influence of process parameters on tailoring the grain morphology of IN718 in electron beam additive manufacturing. Acta Mater. 112, 303314 (2016).

[3] Liu, L., Sommer, F. \& Fu, H. Z. Effect of solidification conditions on MC carbides in a nickel-base superalloy IN 738 LC. Scr. Metall. Mater. (1994) doi:10.1016/0956-716X(94)90434-0. 